Revue des patrimoines

19 | 2012

Patrimoines et conservation préventive. Pratiques comparées et nouveaux enjeux

\title{
Climat et biocontamination. Études de cas d'archives
}

\section{Alain Roche et Sébastien Gilot}

\section{(2) OpenEdition}

Journals

Édition électronique

URL : http://journals.openedition.org/insitu/9788

DOI : $10.4000 /$ insitu.9788

ISSN : 1630-7305

Éditeur

Ministère de la culture

\section{Référence électronique}

Alain Roche et Sébastien Gilot, "Climat et biocontamination. Études de cas d'archives », In Situ [En

ligne], 19 | 2012, mis en ligne le 18 septembre 2012, consulté le 19 avril 2019. URL : http://

journals.openedition.org/insitu/9788 ; DOI : 10.4000/insitu.9788

Ce document a été généré automatiquement le 19 avril 2019

\section{cc)}

In Situ Revues des patrimoines est mis à disposition selon les termes de la licence Creative Commons Attribution - Pas d'Utilisation Commerciale - Pas de Modification 4.0 International. 


\title{
Climat et biocontamination. Études de cas d'archives
}

\author{
Alain Roche et Sébastien Gilot
}

\section{Introduction}

1 La conservation des archives impose des conditions climatiques rigoureuses. Certains matériaux conservés nécessitent des conditions différentes et se trouvent isolés dans des magasins spéciaux. Pour les documents reliés, les archives conservées en boîtes, les journaux, les revues, etc., la Bibliothèque Nationale et les Règles de base pour la construction et l'aménagement d'un bâtiment d'archives, (1999) du service interministériel des Archives de France, recommandent des conditions qui nécessitent pour les maintenir des moyens techniques importants qui ne sont pas à l'abri de dysfonctionnements ou de pannes. Le contenu en documents papier de ces magasins intervient largement dans la régulation du climat.

2 On constate la plupart du temps que les contaminations microbiologiques sont le fait d'une mauvaise conception, d'un dysfonctionnement ou d'une panne du système de régulation de l'air, mais aussi d'un mauvais état du bâti. En effet quelle qu'en soit la cause, c'est avant tout l'augmentation de l'humidité des locaux qui entraîne l'apparition de développements fongiques.

Les moisissures sont des organismes largement répandus dans notre environnement et il est donc naturel d'en retrouver une fraction dans les milieux intérieurs. Le cycle de développement d'une moisissure commence par la germination d'une conidie (ou spore) germinant et produisant un hyphe qui va croître et aboutir à la formation d'un mycélium. À maturité, le mycélium produit des hyphes différenciés dont le rôle sera la production de nouvelles conidies. La conidie assure donc la dispersion des espèces fongiques et représente le principal agent de la contamination d'un bâtiment d'archives. Son entrée et sa dissémination dans le milieu intérieur se fait principalement par l'intermédiaire de mouvements d'air, mais aussi de personnel et de documents d'archives. 

ambiante pour limiter, voire inhiber, les développements fongiques.

\section{Climat produit par un système de traitement de l'air}

\section{Installations techniques et distribution de l'air dans les magasins}

17 Schématiquement, les systèmes de climatisation sont composés de trois éléments principaux : une batterie froide, une batterie chaude et un humidificateur. L'air traité est distribué et récupéré dans les magasins par l'intermédiaire d'un circuit de gaines. Dans chaque magasin, l'air arrive par des bouches de soufflage et l'air vicié est évacué par des bouches de reprise d'air.

Dans d'autres types d'installation et notamment aux Archives départementales du Finistère, Quimper, l'air est soufflé par une bouche équipée d'une batterie à eau chaude et 
récupéré par une ou deux bouches de reprise d'air, située en position opposée dans le magasin. Ces centrales de traitement d'air sont pilotées par un ensemble de régulateurs (température et hygrométrie de l'air soufflé) asservi à des sondes thermo hygrométriques. Pour réguler le climat il est donc possible d'agir sur trois paramètres : la température, la quantité de vapeur d'eau et le débit d'air.

\section{Consignes et zone de tolérance}

Les consignes sont des écarts de valeurs d'humidité et de températures que l'on impose au système de traitement de l'air via les régulateurs. Ces valeurs sont librement prises par les responsables de l'établissement des archives et on peut en principe les modifier à tout moment. Pour une bonne conservation des archives, la Bibliothèque Nationale recommande une zone de tolérance en humidité de $55 \% \pm 5 \%$ et en température $18^{\circ} \mathrm{C} \pm 2^{\circ}$ C.

Le strict respect de ces consignes dépend de la fiabilité et de l'état de fonctionnement du système de traitement de l'air. Le respect des valeurs de consignes est d'autant plus aisé que les écarts autorisés d'humidité et de température sont importants.

«La température doit être comprise entre $16^{\circ} \mathrm{C}$ et $22 / 23^{\circ}$ voire $25^{\circ}$ exceptionnellement avec une variation maxima de $2^{\circ} \mathrm{C}$ par semaine et $\mathrm{d}^{\prime} 1^{\circ}$ par 24 heures.

L'hygrométrie à corréler avec la température doit être comprise entre 45 et $55 \%$ maxima pour éviter le développement de microorganismes. Une variation maxima de $5 \%$ d'humidité relative par jour est admise.»

21 Remarque ; le SIAF a assoupli ces consignes en 1999:

22 Un contrôle climatique indépendant des sondes thermo hygrométriques de régulation est en mesure de détecter les dérives de fonctionnement d'un système de traitement de l'air. Nous avons pu remarquer qu'aux archives de Quimper, établissement équipé d'un système de climatisation, les valeurs d'humidité relative et de température de certains magasins correspondaient à plus de $80 \%$ aux consignes : température comprise entre $16^{\circ}$ $\mathrm{C}$ et $22^{\circ} \mathrm{C}$, et une humidité relative comprise entre $45 \%$ et $60 \%$. En revanche, dans d'autres salles, le pourcentage de valeurs respectant les mêmes consignes pouvaient être beaucoup plus faibles, indiquant ainsi la présence de problèmes.

Deux exemples d'analyse d'événements nous permettront de mieux comprendre les difficultés de régulation de l'air.

Les courbes d'humidité relative, de température et de teneur en eau (fig. $\mathbf{n}^{\circ} \mathbf{1}$ ) décrivent le climat de l'un des magasins des archives de Quimper pendant la durée de la campagne de mesures. On remarque que les valeurs de températures sont hors des zones de recommandation strictes de la BnF alors que l'humidité reste dans les limites admises. Analysons l'événement qui s'est produit entre les heures 519 et 521. 


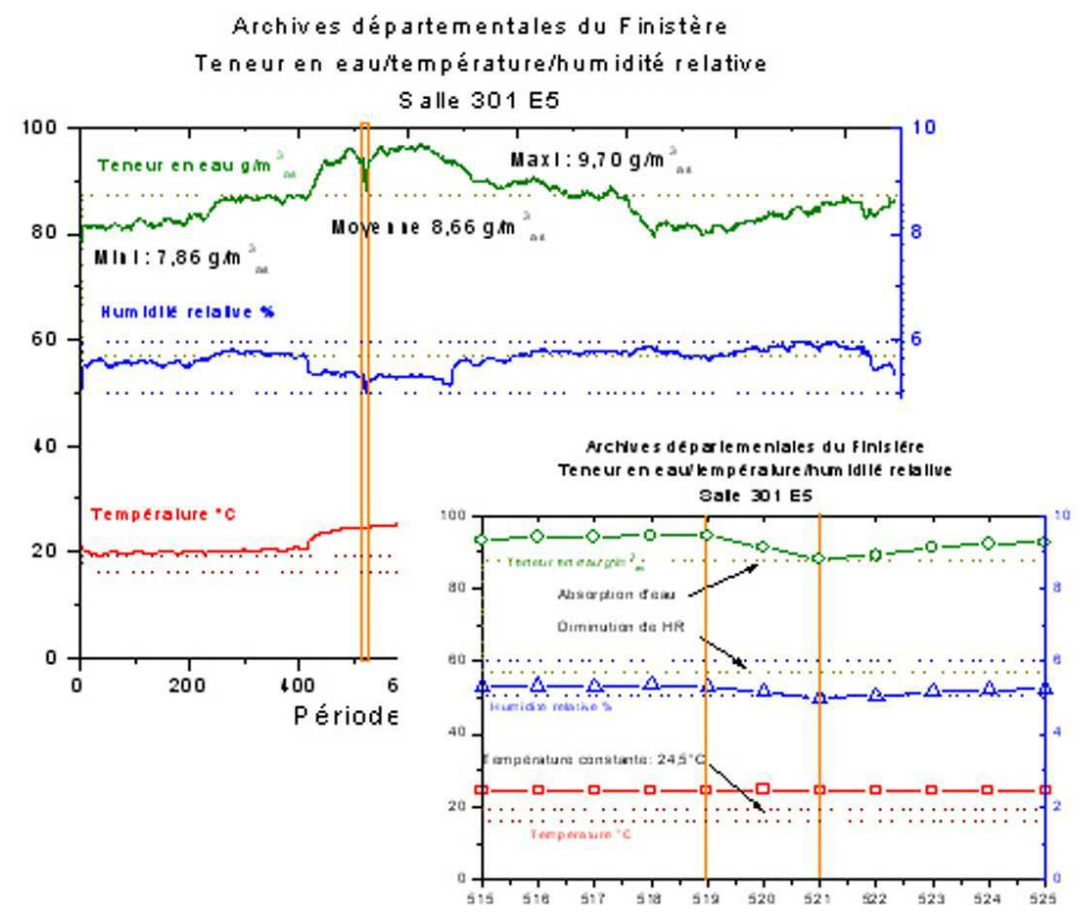

Étude climatique Archives départementales du Finistère, absorption d'eau.

(c) Alain Roche.

On constate que pendant ces deux heures la température est constante. Elle est de $24,5^{\circ} \mathrm{C}$ et se situe au-dessus de la limite supérieure de recommandation. Néanmoins pendant ces deux heures l'humidité relative diminue, en passant de $53,1 \%$ à $49,8 \%$, soit un écart de $3,3 \%$. Ce phénomène ne peut s'expliquer que par une absorption de la vapeur d'eau contenue dans l'air de cette salle, attestée par la chute des valeurs de vapeur d'eau. Quelle qu'en soit la cause, le système de traitement d'air devra réagir en rétablissant le taux d'humidité nécessaire.

Cette sensibilité du climat à de petites variations et les difficultés de régulation sont illustrées par un autre type d'événement (fig. $\mathbf{n}^{\circ} 2$ ). 


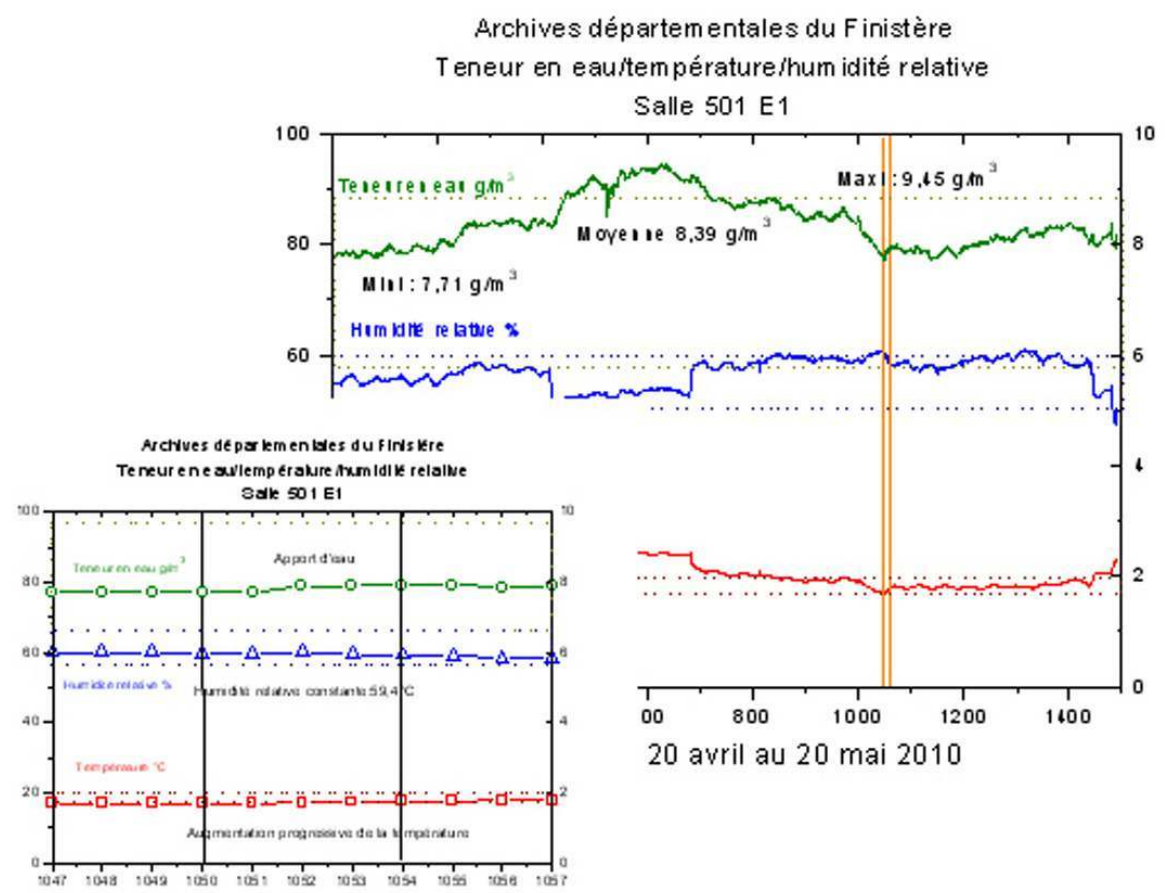

Étude climatique Archives départementales du Finistère, apport d'eau.

(c) Alain Roche.

Entre les heures 1050 et 1054, l'humidité relative reste constante à 59,4\% alors que la température augmente progressivement de $16,9^{\circ} \mathrm{C}$ à $17,6^{\circ} \mathrm{C}$. D'après la définition de l'humidité relative, si la température s'élève, le degré de saturation $x_{s(T)}$ augmente et l'humidité relative devrait diminuer à moins qu'un apport d'eau extérieur modifie $x$ de telle manière que l'humidité relative reste constante.

Bien entendu, il est souvent difficile d'identifier l'origine de l'absorption ou de l'apport des vapeurs d'eau. Les systèmes de régulation du climat ont du mal à gérer ces événements de courte durée et dans ce cas les valeurs d'humidité et de température peuvent aisément sortir de la zone de tolérance.

\section{Climat produit par une VMC double flux}

\section{Installation technique et distribution de l'air dans les magasins}

La ventilation mécanique contrôlée (VMC) double flux distribue un air filtré extrait généralement de l'extérieur comme dans le cas des archives municipales de Boulognesur-Mer. Les magasins sont alimentés en air par l'intermédiaire d'un réseau de gaines percées de bouches de soufflage. Ce type d'installation ne permet pas d'agir sur la qualité de l'air en termes d'humidité et de température, mais d'obtenir un renouvellement. Les seuls paramètres sur lesquels on peut intervenir sont le débit d'air et une augmentation de la température par un apport extérieur de chaleur. 


\section{Influence du climat extérieur}

Dans l'exemple suivant, la superposition de la courbe d'humidité relative du climat extérieur aux courbes d'humidité relative d'une salle des archives de Boulogne-sur-Mer (fig. $\mathbf{n}^{\circ} \mathbf{3}$ ) souligne la similitude du comportement des climats, pendant la période allant d'août à mi-octobre. Par contre, dès que la salle est chauffée, les courbes se différencient sans que l'influence du climat extérieur cesse d'agir sur le climat de la salle. Le papier contenu dans les archives est hygroscopique, c'est-à-dire qu'il absorbe et désorbe de l'eau de manière à être toujours en équilibre hydrique avec son milieu climatique. En grande quantité, la présence de ce papier va amortir les variations d'humidité dans les magasins. Cet effet tampon est perceptible par exemple dans ce suivi entre les heures 950 et 1150, mais en aucun cas les archives sont chargées de réguler le climat des salles.

Figure 3
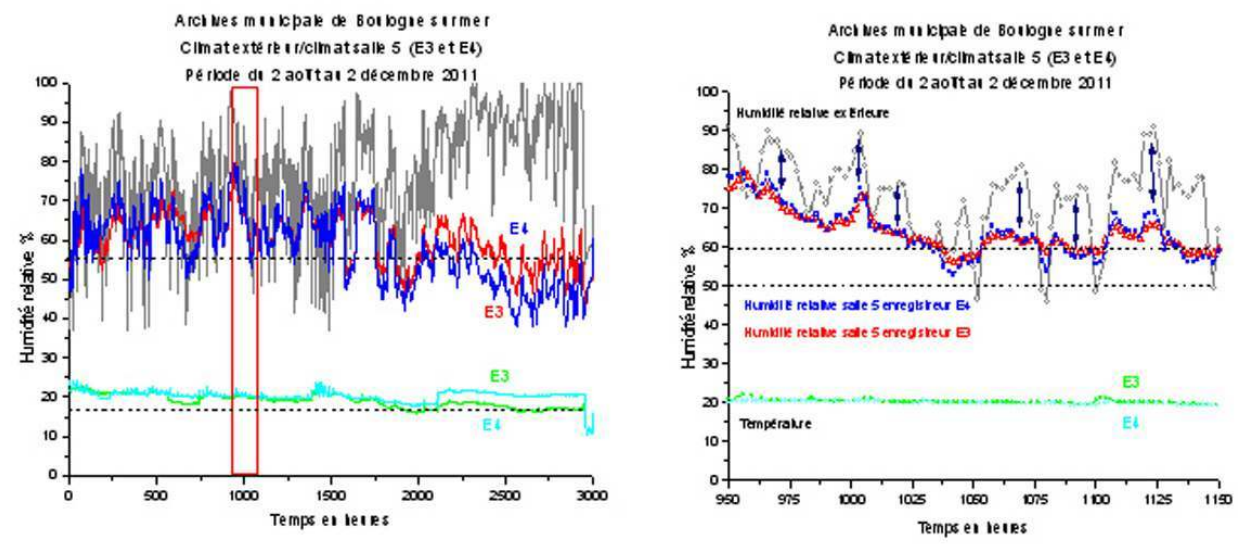

Étude climatique, Archives municipale de Boulogne-sur-Mer, influence du climat extérieur.

(c) Alain Roche.

Si l'humidité extérieure est élevée, par voie de conséquence, elle peut être responsable d'une importante humidité relative à l'intérieur des salles. Pendant les 36 heures de relevés entre les heures 920 et 956, l'humidité moyenne de la salle 5 a atteint 77,45\% et elle se trouve à la limite des valeurs d'activité en eau de certaines espèces.

Cet état est inhérent aux installations VMC qui ne permettent pas d'imposer de consignes et le climat qui règne dans les salles est aléatoire et relativement instable.

\section{Rôle des boîtes d'archives vis-à-vis des documents}

La première barrière aux variations climatiques extérieures est le bâtiment qui naturellement temporise les écarts. À l'intérieur des magasins, une grande partie des documents est conservée dans des boîtes d'archives, généralement conçues en carton compact et répondant aux normes ISO 16245. Bien que son rôle soit de protéger les documents de la poussière, elle joue également un rôle de barrière à l'humidité. L'exemple pris aux archives de Boulogne-sur-Mer illustre bien la fonction jouée par la boîte et son contenu vis-à-vis de l'humidité et de la température (fig. $\mathbf{n}^{\circ} \mathbf{4}$ ). 



Étude climatique, Archives municipale de Boulogne-sur-Mer, effet tampon des boîtes d'archives. (c) Alain Roche.

À l'extérieur de la boîte, pendant les 500 heures d'enregistrement, nous avons une série de variations d'humidité supérieures à $10 \%$ et un écart maximum de $29,6 \%$. Par contre à l'intérieur de la boîte, il n'y a plus de pics de variations d'humidité et l'écart maximum est réduit à $6 \%$. La boîte et son contenu ont par conséquent un effet régulateur évident, aussi bien au niveau hydrique que thermique. Dans ce processus, ne connaissant pas la fraction d'eau absorbée par la boîte, on est amené à penser que le contenu de la boîte joue également un rôle et que cet effet tampon exerce sur les documents des contraintes d'ordres physique et mécanique.

\section{Évaluation des niveaux de contamination}

\section{Évaluations visuelles}

L'évaluation du niveau de biocontamination de magasins d'archives intervient généralement après l'identification visuelle, par le personnel des archives, de développements fongiques sur les documents qui y sont conservés.

À première vue, les situations fongiques au sein de bâtiments d'archives apparaissent peu variées: les contaminations visuellement identifiées peuvent être discontinues et dispersées dans les différents magasins ou être concentrées dans quelques zones. Du fait de leur plus grande exposition à l'environnement, les développements fongiques sont le plus couramment observés sur les couvertures, les tranches des ouvrages et des liasses 
ainsi que sur les conditionnements. Entre autres facteurs, la qualité et la nature des matériaux influent sur la répartition des colonies dans les magasins.

À Quimper par exemple, les magasins peuvent être aisément classés en fonction du rapport zones visuellement contaminées / zones visuellement saines :

$38-2 / 5^{\text {èmes }}$ sont caractérisés par une contamination importante ;

$39-2 / 5^{\text {èmes }}$ par une contamination faible ;

$40-1 / 5^{\text {ème }}$ par une contamination non caractérisée du fait de forts dépôts et empoussièrement.

41 Les documents les plus touchés sont les reliures toilées, cuirs et boîtes d'archives anciennes.

42 À Boulogne-sur-Mer en revanche, les documents visuellement contaminés sont disséminés dans les magasins sans que n'émerge une répartition préférentielle. Les documents les plus touchés sont les reliures toilées et les bandes toilées des chemises d'archives. S'ajoutent de façon plus ponctuelle des ouvrages reliés en cuir.

43 Les densités de mycélium observées, leurs fréquences et leurs concentrations sur documents sont plus importantes à Quimper qu'à Boulogne-sur-Mer.

44 Ces premières observations permettent de définir l'échantillonnage proposé qui doit conduire à la sélection de points de prélèvement significatifs. Leur nombre et leur variété définissent le niveau d'analyse que l'on peut espérer atteindre en permettant les comparaisons tant quantitatives que qualitatives. L'échantillonnage doit donc être adapté à la configuration des contaminations et des bâtiments d'archives. Il doit permettre de vérifier le caractère actif ou non de la biocontamination, de la quantifier, d'identifier les contaminants présents afin d'en évaluer l'impact vis-à-vis des collections et enfin d'identifier au mieux les sources et les facteurs de développement.

Il est donc important qu'un maximum d'éléments soit pris en compte :

46 - documents (visuellement contaminés ou non);

47 - environnement intérieur (surfaces de stockage, murs, sols, étagères, air ambiant, système de ventilation) et extérieur (le plus souvent air) ;

48 - climat.

De l'analyse de chacun de ces éléments et de leur confrontation peut émerger un schéma.

\section{Mises en culture : documents}

50 L'évaluation quantitative de la biocontamination s'effectue par le comptage des colonies se développant sur les milieux de cultures. Elle est exprimée en $\mathrm{UFC} / \mathrm{cm}^{2}$ (Unité Formant Colonie).

51 Les résultats de mise en culture peuvent permettre, selon les cas, de confirmer et préciser les observations visuelles, en relativiser l'importance ou mettre en lumière des problèmes sous-jacents. On peut en effet s'attendre, à priori, à ce que des documents visuellement contaminés présentent en culture des densités de colonies supérieures à celles de documents visuellement sains et à celles relevées dans leur environnement. Ce n'est pas systématiquement le cas.

52 Ainsi dans notre exemple, la présence de colonies à Quimper est importante en termes de surface touchée. Néanmoins, il n'est pas possible d'établir de corrélation entre densité de 
reviviscence ${ }^{1}$ et présence de colonies sur les documents. Les concentrations en espèces reviviscibles sur documents présentent une grande homogénéité au travers des magasins échantillonnés. $90 \%$ des écouvillons réalisés montrent des densités $\leq 10 \mathrm{UFC} / \mathrm{cm}^{2}$, ce qui est très faible, que les prélèvements aient été réalisés sur documents visuellement contaminés ou non.

De même, aucune différence significative n'apparaît entre les échantillons prélevés sur des surfaces exposées à l'environnement et sur des surfaces protégées (documents conditionnés). Seule la salle des microfilms présente une telle répartition. Les documents conservés en meubles à plans sont plus faiblement contaminés que les documents conservés sur les meubles ( $70 \%$ de ces derniers présentent des densités comprises entre 50 et $\left.70 \mathrm{UFC} / \mathrm{cm}^{2}\right)$.

54 À Boulogne-sur-Mer en revanche, les concentrations en espèces reviviscibles sur documents permettent de les classer en deux catégories :

55 - Densité $\leq 20 \mathrm{UFC} / \mathrm{cm}^{2}$. Cette catégorie se compose de la totalité des échantillons visuellement non contaminés et collectés en corps d'ouvrage et à l'intérieur des boîtes d'archives et d'un seul échantillon contaminé.

56 - Densité $>20 \mathrm{UFC} / \mathrm{cm}^{2}$ : regroupant à $85 \%$ des documents visuellement contaminés (max à $66 \mathrm{UFC} / \mathrm{cm}^{2}$ ) et à $90 \%$ des prélèvements sur surfaces exposées à l'environnement. Les documents visuellement non contaminés présentent les UFC minimales de la catégorie.

57 Dans ce cas, la densité de reviviscence est de façon générale corrélable à la présence de colonies visuellement identifiables sur documents et à la présence ou non d'un conditionnement. Cependant, les niveaux de contaminations relevés restent faibles.

\section{Mises en culture : environnement}

58 Au moment de l'analyse des données, l'échantillonnage réalisé en environnement permet de contextualiser les observations concernant les niveaux de reviviscence relevés sur documents. Judicieusement choisi, il permet notamment d'identifier des zones préférentielles d'entrée, de sédimentation et/ou de conservation des conidies.

59 Si un système de traitement de l'air est présent, l'échantillonnage permet d'évaluer son rôle. La climatisation peut agir comme un agent de dispersion, un initiateur du développement, une source et réservoir de contaminants.

60 À Quimper, et à l'inverse de ce que l'on peut observer sur documents, les échantillons prélevés en environnement présentent des profils légèrement différents entre les magasins faiblement contaminés et les magasins fortement contaminés. Dans les premiers, on rencontre des niveaux de reviviscence faibles (murs $<10 \mathrm{UFC} / \mathrm{cm}^{2}$ et majorité des étagères $<20 \mathrm{UFC} / \mathrm{cm}^{2}$ ). Dans les seconds, les niveaux de reviviscences sur murs deviennent supérieurs à $10 \mathrm{UFC} / \mathrm{cm}^{2}$ et sur les étagères majoritairement supérieurs à $20 \mathrm{UFC} / \mathrm{cm}^{2}$. À nouveau, la salle des microfilms se singularise avec des niveaux relevés de $38 \mathrm{UFC} / \mathrm{cm}^{2}$ sur les murs et $182 \mathrm{UFC} / \mathrm{cm}^{2}$ sur les meubles. Quelques zones de plus forte densité apparaissent également dans les magasins fortement contaminés au niveau des huisseries qui peuvent définir des points d'entrée de contaminants fongiques et dans les zones relativement protégées du flux d'air issu du système de ventilation.

61 De façon générale, les densités en espèces reviviscibles sont plus élevées en environnement que sur documents. 
62 Au niveau du système de climatisation, il faut noter que pour les centrales de traitement d'air (CTA) alimentant les grands magasins, les caissons de ventilation ont été nettoyés peu de temps avant la campagne de prélèvement. Ce n'est pas le cas de la CTA des petites salles où une forte densité fongique reviviscible est observée dans le caisson de ventilation après filtration. La filtration est inopérante et cette CTA agit comme un agent de dispersion et de renouvellement de la biocontamination dans les salles qu'elle distribue.

63 La comparaison des taux de reviviscence entre arrivées et en reprises d'air montre un enrichissement en espèces reviviscibles dans tous les magasins. Alors que les densités au niveau des bouches d'arrivée d'air sont équivalentes à celles relevées sur les étagères, les densités relevées aux bouches de reprise d'air y sont deux fois plus élevées.

Du point de vue de la répartition des spores, l'analyse de la biocontamination démontre que plus le point de prélèvement est éloigné du flux d'air plus l'air se trouve chargé en spores. De plus, les niveaux les plus élevés de contamination de l'air sont relevés dans les magasins les plus fortement contaminés.

À Boulogne-sur-Mer, les échantillons prélevés en environnement présentent un profil de reviviscence homogène au travers des magasins échantillonnés (murs $<10 \mathrm{UFC} / \mathrm{cm}^{2}$ et majorité des étagères $<20 \mathrm{UFC} / \mathrm{cm}^{2}$ ) équivalent à celui relevé à Quimper. La seule exception relevée est la salle de déchargement où on trouve des densités de reviviscence beaucoup plus élevées (avec $145 \mathrm{UFC} / \mathrm{cm}^{2}$ sur les murs, mais une densité sur les étagères hautes équivalent au reste du bâtiment).

De façon générale, et à l'exception de cette salle, les densités en espèces reviviscibles sont plus élevées sur les documents qu'en environnement.

67 Aucune corrélation n'a pu être mise en évidence entre niveau de contamination en magasins et en système d'extraction de l'air (ou les niveaux de reviviscence sont relativement constants tout au long des gaines et équivalents entre bouches d'arrivée d'air et bouches de reprise d'air).

En revanche, l'analyse de la biocontamination de l'air montre clairement un gradient dans la répartition des contaminants fongiques aéroportés entre les magasins proches de l'entrée de la salle de déchargement et les magasins qui en sont les plus éloignés (en suivant ce déplacement, l'air intérieur contient, aux points d'échantillons $1268 \mathrm{UFC} / \mathrm{m}^{3}$, $142 \mathrm{UFC} / \mathrm{m}^{3}$ pour finir à $44 \mathrm{UFC} / \mathrm{m}^{3}$ ). Ces valeurs correspondent, pour les environnements de travail, respectivement, à des contaminations de l'air élevée, moyenne et faible.

Même si le gradient de contamination identifié dans l'air ne se retrouve ni sur les documents ni sur les surfaces dans les magasins échantillonnés, il n'en reste pas moins qu'il existe dans le bâtiment une large voie d'entrée de contaminants fongiques depuis l'extérieur.

\section{Conclusion}

70 Dans les magasins d'archives, parmi les documents les plus divers, les organismes fongiques attendent patiemment que les conditions climatiques leurs soient favorables pour éclore. C'est pour cette raison que le maintien d'un climat sec et frais est indispensable à la bonne conservation des archives. Dans le cas d'une alerte, l'évaluation de la situation microbiologique n'est pas, à elle seule, suffisante. Les caractéristiques 
fongiques exposées au travers des exemples de Quimper et de Boulogne-sur-Mer pourraient être relevées dans un bâtiment d'archives sain, dans lequel on ne ferait que relever un risque plus ou moins élevé de développement en fonction des densités et de la nature des contaminants identifiés. Cependant, ce sont les caractéristiques climatiques du bâtiment qui vont transformer un risque en réalité. Le diagnostic et le contrôle corrects de la qualité de l'air des magasins nécessitent une étude climatique complétant l'étude de biocontamination.

71 Si l'on veut éviter qu'un départ de développement fongique ne devienne critique, une telle étude doit être décidée rapidement afin de permettre la mise en place de mesures correctives adaptées à la situation du bâtiment.

72 À Quimper nous avons pu mettre en relation une défaillance du système de traitement de l'air avec une concentration en spores reviviscibles plus importante en environnement que sur documents. Les analyses quantitative et qualitative de la biocontamination montrent que les densités en spores reviviscibles sont faibles et que les espèces fongiques présentes sont peu variées et fortement dispersées dans les magasins. Au vu de la forte présence de colonies fongiques sur les documents, cette répartition est inexplicable sans une étude du climat et des caractéristiques techniques du système de traitement de l'air. les CTA ont ramené le climat intérieur dans un relatif respect des consignes. Mais ces interventions ne tiennent compte ni de l'état et de la réponse du bâti aux variations climatiques extérieures, ni des différents comportements des magasins à ces variations. Malgré les efforts accomplis, le système de régulation est instable et de nouveaux dérèglements sont à prévoir.

75 - Le taux de renouvellement de l'air est excessivement élevé (8 volumes par heure). C'est cette caractéristique de l'installation technique qui permet essentiellement d'expliquer la contradiction apparente entre la présence de nombreuses colonies dans le bâtiment et les faibles concentrations en culture. Avec le temps, le fort mouvement d'air dans les magasins a eu un effet positif en réduisant la présence de conidies reviviscibles sur les documents mais a aussi eu un effet négatif en favorisant leur dispersion.

Les genres fongiques isolés dans les magasins de Quimper sont très majoritairement Penicillium (Aw 0,78), Cladosporium (Aw 0,85), Absidia (Aw 0,88) et Acremonium (Aw 0,90 ). Aux points des plus fortes densités et aux niveaux des huisseries, on relève également la présence des genres Aspergillus (Aw 0,75) et Moniliella (Aw 0,90). En cas d'élévation de l'humidité relative, Aspergillus et Penicillium seront les premiers à se développer.

77

À Boulogne-sur-Mer, c'est le système de ventilation mécanique qui est responsable de l'apparition des développements fongiques. On a déjà noté que ce type d'installation ne permet pas la régulation du climat intérieur et réduit même la capacité du bâtiment à tamponner les écarts thermo hygrométriques extérieurs. Elle aboutit à la création cyclique de conditions favorables puis défavorables à la germination et à la croissance fongiques. La qualité du climat intérieur est soumise aux variations journalières et saisonnières. Ainsi, au moment de l'étude climatique, la mise en fonctionnement du chauffage dans les locaux administratifs a conduit à un abaissement de l'hygrométrie ambiante et à un arrêt des développements fongiques, mais il est certain que ceux-ci reprendront au prochain changement de saison et aussi longtemps que le milieu intérieur 
sera ainsi assujetti au climat extérieur. Les phases de forte humidité peuvent être de courte durée, ce qui explique les faibles taux de reviviscence relevés en culture.

Les espèces fongiques relevées sont plus variées qu'à Quimper et leur répartition plus variable en fonction des localisations dans le bâtiment. Les genres fongiques les plus présents sont Aspergillus (Aw 0,75), Penicillium (Aw 0,78), Paecilomyces (Aw 0,79), Cladosporium (Aw 0,85), Phialophora (Aw 0,85), Fusarium (Aw 0,87), Acremonium (Aw 0,90 ) et Monascus (Aw 0,93).

La flore fongique relevée dans l'air présente des recoupements partiels avec les genres précités notamment Paecilomyces, Cladosporium, Phialophora, Acremonium et Monascus.

Enfin, l'étude climatique des magasins des archives de Boulogne-sur-Mer a montré le rôle joué par les conditionnements dans la minimisation des écarts d'humidité à l'intérieur des boîtes d'archives. Cet effet tampon peut être mesuré en termes de développement fongique :

81 - une très faible proportion des colonies visuellement identifiables a été observée à l'intérieur des boîtes.

82 - les taux de reviviscence mesurés en culture sont systématiquement plus faibles à l'intérieur des boîtes.

83 L'intérêt du conditionnement en milieu d'archives apparait donc double, permettant de lisser les fluctuations d'HR et de réduire le dépôt de spores fongiques sur les documents.

\section{BIBLIOGRAPHIE}

PEDREGAL, Pierre. Climat des magasins d'archives : objectifs, moyens, méthodes. 2009, DAF.

Règles de base pour la construction et l'aménagement d'un bâtiment d'archives. 2009, DAF.

FLIEDER, Françoise, CAPDEROU, Christine. Sauvegarde des collections du patrimoine : la lutte contre les détériorations biologiques. Paris : CNRS, 1999.

ROQUEBERT, Marie-France. Les contaminants biologiques des biens culturels. Paris : Muséum d'histoire naturelle Elsevier, 2002.

GENTY, Guillaume, LAVEDRINE, Bertrand, LECLERC, Brigitte, LE ROY, Sylvie, TILLIER, Annick, ROKOTONIRANY, Malala. La contamination des collections et des locaux des bibliothèques par des moisissures : Méthodes de détection et d'évaluation. Direction du livre et de la lecture.

PARCHAS, Marie-Dominique. Le dépoussiérage des archives et de leur environnement : aide à

l'élaboration d'un cahier des charges. DAF, 2009.

PARCHAS, Marie-Dominique. Conseils en cas de contamination de moisissures. DAF, 2007. 


\section{NOTES}

1. - Faculté de se développer sur milieu de culture.

\section{RÉSUMÉS}

À la suite de problèmes de contamination microbiologique de documents en magasins d'archives, nous avons été mandatés à plusieurs reprises afin d'évaluer les niveaux de biocontamination et de proposer des préconisations et solutions. C'est à partir d'une étude de climat et de biocontamination que nous avons pu, dans la plupart des cas, mettre en relation les situations fongiques observées et leurs causes probables. Nous présentons dans cet article deux cas qui nous ont semblé intéressants et complémentaires: les conséquences des dysfonctionnements des systèmes de traitement de l'air et l'instabilité des climats produits par une VMC à double flux. Même si, dans ces deux cas, les situations fongiques apparaissent identiques, les causes de développement peuvent être très différentes. Il est donc essentiel de remonter aux origines des dysfonctionnements et des déséquilibres climatiques. L'étude microbiologique fait état des niveaux de contamination en espèces reviviscibles sur les documents, en environnement et dans l'air ambiant. Elle localise les zones de forte concentration qu'elle relie directement aux données micro climatiques des magasins, analysées parallèlement. C'est donc en s'appuyant sur l'étude des microclimats, sur l'analyse des événements climatiques dans les archives et des conditions climatiques environnementales que l'on peut espérer déterminer les facteurs de contamination.

Following problems of microbiological contamination of documents in archives, we were repeatedly appointed to evaluate the biocontamination levels and propose recommendations and solutions. It is from the study of both climate and biocontamination that we were able, in most of the cases, to relate the observed fungal situations to their likely causes. We present in this article two cases which seemed to us interesting and complementary: the consequences of dysfunctions of an air handling system and the instability of climates produced by a Mechanical Extract Ventilation system. Even if the fungal situations seem identical, causes of fungal growth can vary greatly. Thus, it is essential to go back up to the origins of the dysfunctions and climate instabilities. The microbiological study states levels of viable species on documents, in environment and in the ambient air. It localizes the areas of strong concentration that it connects directly to the microclimate data collected in the storerooms. Thus while leaning on the study of the microclimates, on the analysis of climate-related events in archives and environmental weather conditions one can hope to determine the factors of contamination.

\section{INDEX}

Mots-clés : archives, contamination fongique, climats, traitement de l'air, ventilation. 


\section{AUTEURS}

\section{ALAIN ROCHE}

Laboratoire d'analyse et de recherche pour la conservation et la restauration d'œuvres d'art (LARCROA) a.roche@larcroa.fr

\section{SÉBASTIEN GILOT}

Laboratoire d'analyse et de recherche pour la conservation et la restauration d'œuvres d'art (LARCROA) Sebgil@wanadoo.fr 